\title{
The Arcuate Fasciculus and Language Development in a Cohort of Pediatric Patients with Malformations of Cortical Development
}

\author{
M.J. Paldino, K. Hedges, and (D)F. Golriz
}

\begin{abstract}
BACKGROUND AND PURPOSE: Patients with epilepsy and malformations of cortical development have a high prevalence of language deficits. The purpose of this study was to investigate whether the status of the arcuate fasciculus at diffusion tractography could provide a clinically meaningful marker of language function in patients with cortical malformations.
\end{abstract}

MATERIALS AND METHODS: Thirty-seven patients 3-18 years of age who had DTI performed at 3T and language evaluation by a pediatric neurologist were retrospectively identified. Twenty-two age-matched children without any neurologic, language, or MR imaging abnormalities who had identical DTI performed for an indication of headache were selected as a control cohort. The arcuate fasciculi were constructed and segmented by deterministic tractography for all subjects.

RESULTS: Twenty-one patients had intact language; 11 had mild-to-moderate and 5, profound language impairment. All patients with normal language and all control subjects had an identifiable left arcuate. The left arcuate was absent in 11 patients; all 11 were languageimpaired. Failure to identify the left arcuate was strongly associated with some degree of language impairment $(P<.001)$. Sensitivity, specificity, and positive predictive value for language dysfunction were $65 \%, 100 \%$, and $100 \%$, respectively. The absence of the arcuate bilaterally was associated with complete failure to develop oral language $(P<.015)$.

CONCLUSIONS: Failure to identify the left arcuate fasciculus at diffusion tractography was a highly specific marker of language dysfunction in a cohort of pediatric patients with malformations of cortical development. Failure to identify the arcuate fasciculus on either side was associated with failure to develop oral language.

ABBREVIATION: MCD = malformation of cortical development

M ost higher order functions of the human brain are not accomplished by individual functional centers compartmentalized to a particular region of the cortex. Rather, they emerge from parallel processing within subspecialized, but distributed, functional systems. A complex neural network, formed by some 10 billion neurons, forms the structural substrate for efficient interaction between local and distributed areas of the cerebrum. Diffusion tractography is an extension of DTI, which uses the directional tendencies of water diffusion to construct 3D trajectories of white matter tracts based on their structural coherence. ${ }^{1-3}$ The functional importance of many such fiber systems has now been described; this description has allowed assessment

Received March 6, 2015; accepted after revision May 12.

From the Department of Radiology (M.J.P., K.H.), Children's Hospital Boston and Harvard Medical School, Boston, Massachusetts; and Department of Radiology (F.G.), Texas Children's Hospital, Houston, Texas.

Please address correspondence to Farahnaz Golriz, MD, Department of Radiology, Texas Children's Hospital, 6701 Fannin St, Houston, TX 77030; e-mail: fxgolriz@ texaschildrens.org

http://dx.doi.org/10.3174/ajnr.A4461 of brain white matter abnormalities in terms of functional systems. ${ }^{4,5}$ Despite the obvious promise of this technique, the ability to apply quantitative information derived from DTI toward management of an individual patient has, to date, proved elusive.

Epilepsy is a common neurologic condition defined by recurrent, unprovoked seizures, which affects $1 \%$ of the population, including 1 in 200 children. ${ }^{6,7}$ Unlike in adults, developmental lesions predominate as the source of seizures in children; in particular, malformations of cortical development (MCDs) are the most common anatomic substrate for intractable epilepsy in children. ${ }^{8}$ Patients with epilepsy and MCDs are at high risk for language and other cognitive impairments. Although the work-up has traditionally centered on detecting localized dysplastic abnormalities, patients with MCDs experience a wide range of deficits, which often cannot be explained on the basis of the location of the structural abnormality. ${ }^{9-13}$ The cortical structure outside the region of MCD is normal, at least in the sense that it does not demonstrate the same histopathologic abnormalities that characterize a dysplastic cortex. Therefore, the occurrence of such a wide 
range of functional abnormalities implies the importance of aberrant cortico-cortical interaction or connectivity. Regardless of whether such aberrant connectivity is established by genetic/developmental processes or by activity-dependent reorganization, and there is evidence to support a role for each, such abnormalities have obvious potential implications for neurocognitive development. ${ }^{14-19}$

Recent work has demonstrated the potential for machine learning to translate quantitative data from whole-brain tractography into phenotypic information regarding language function in an individual patient. ${ }^{20}$ Although highly accurate, this technique is time-consuming and requires substantial expertise in image processing and mathematics/statistics. These issues constitute a barrier to widespread adoption of such approaches into clinical practice, especially outside academic centers. There is a need for more practical imaging markers of language dysfunction.

The arcuate fasciculus is a major intrahemispheric association pathway that connects important receptive and expressive speech areas in the frontal and temporal lobes. ${ }^{4,21}$ Although the exact roles of this pathway remain the subject of debate, strong evidence suggests an important contribution to language function. ${ }^{22,23}$ Furthermore, the absence of the arcuate fasciculus has been reported in several neurodevelopmental disorders that manifest language impairment. ${ }^{24}$ Together with recent work demonstrating that absence of the arcuate fasciculus at diffusion tractography is a highly reproducible binary finding, these results make the arcuate a very attractive target as a potential imaging marker of language function. ${ }^{25}$ Hence, the goal of this study was to define the relationship between the arcuate fasciculi at diffusion tractography and the function of the language network in pediatric patients with MCD.

\section{MATERIALS AND METHODS}

This Health Insurance Portability and Accountability Act-compliant study was approved by the local institutional review board. Patients were identified retrospectively with the following inclusion criteria: 1) pediatric age group (18 years of age or younger); 2) diagnosis of a MCD established by MR imaging; 3) MR imaging of the brain performed at 3T, including DTI; and 4) language development characterized by a pediatric neurologist, within 1 month of the above brain imaging examination. Refinements to the above-defined population were based on the following exclusion criteria: 1) motion or other degradation to image quality, and 2) exclusion of patients younger than 3 years of age to increase confidence in the clinical determination of language function.

An age- and sex-matched control group was also retrospectively identified with the following inclusion criteria: 1) clinical indication of headache; 2) MR imaging of the brain performed at 3T, including DTI (identical to DTI performed in the patient group); and 3) language characterized as normal by a pediatric neurologist. Exclusion criteria were the following: 1) any neurologic abnormality by history or physical examination, 2) any degree of language impairment, 3) any MR imaging abnormality, and 4) marked motion or other degradation to image quality.

Patients were divided into 3 groups based on characterization of their language development by a pediatric neurologist:
1) intact: age-appropriate; 2) mild-to-moderate impairment: delayed by comparison with peers (either expressive or receptive); and 3) profound impairment: absent oral language. This categorization was made retrospectively at the time of this study on the basis of the subjective assessment made by a pediatric neurologist at the time of clinical examination. This 3-point scale was selected because it has been used to provide both a clinically meaningful and reproducible estimate of language function. ${ }^{26}$

\section{MR Imaging}

All imaging was performed on two 3T magnets (2 Tim Trio; Siemens, Erlangen, Germany). We performed the following sequences: 1) sagittal magnetization-prepared rapid acquisition of gradient echo (TR/TE, $2530 / 3.39 \mathrm{~ms}$; 1 acquisition; flip angle, $7^{\circ}$; TI, $1100 \mathrm{~ms}$; acceleration, 2; voxel size, $1 \times 1 \times 1 \mathrm{~mm}$ ); 2) axial fast spin-echo T2-weighted (TR/TE, 11,730/89 ms; 2 acquisitions; flip angle, $120^{\circ}$; acceleration, 2 ; voxel size, $0.6 \times 0.4 \times 2.5 \mathrm{~mm}$ ); 3 ) axial fluid-attenuated inversion recovery (TR/TE, 9000/137 ms; 1 acquisition; flip angle, $150^{\circ}$; FOV, $22 \mathrm{~cm}$; voxel size, $0.7 \times 0.7 \times 4$ $\mathrm{mm}$ ); and 4) axial single-shot echo-planar imaging DTI (TR/TE, 7000/90 ms; flip angle, $90^{\circ}$; 1 acquisition; voxel size, $2 \times 2 \times 2$ $\mathrm{mm})$. For DTI, 35 image sets were acquired, 5 without diffusion weighting (B0) and 30 with noncollinear diffusion-weighting gradients $\left(b=1000 \mathrm{~s} / \mathrm{mm}^{2}\right)$. All images were visually inspected for artifacts, including subject motion.

\section{Image Processing and Analysis}

Maps of mean diffusivity and fractional anisotropy were created by using the Diffusion Toolkit (www.Trackvis.org/dtk). For each voxel, a tensor matrix was derived. After diagonalization of the matrix, we obtained eigenvalues, and mean diffusivity and fractional anisotropy were quantified for each pixel according to standard equations. ${ }^{27}$ Two users experienced in tractography performed tract reconstruction, segmentation, and analysis. The Diffusion Toolkit was used for deterministic tract reconstruction by using a fiber association by continuous tracking algorithm $\left(35^{\circ}\right.$ angular threshold). A DWI mask was used to remove CSF, a process that has been shown to effectively prevent spurious tract reconstruction. ${ }^{5}$ TrackVis (www.trackvis.org) was then used for segmentation and analysis of the arcuate fasciculus. ROIs for tract segmentation were placed manually on the color fractional anisotropy maps cross-referenced to the B0 images according to previously described methods. ${ }^{28}$ The arcuate fasciculus in each subject was categorized as present on the left only, right only, or bilaterally. This method has been shown previously to provide a highly reliable classification of the arcuate fasciculus with respect to its absence versus presence. ${ }^{25}$ Mean fractional anisotropy and mean diffusivity were then calculated for each identifiable arcuate fasciculus.

\section{Statistics}

Statistical testing was performed by using SAS software, Version 9.2 (SAS Institute, Cary, North Carolina). Proportions of subjects with-versus-without the absence of the arcuate fasciculus and with-versus-without language impairment were compared by using the Fisher exact test $(\alpha=.05)$. The Wilcoxon rank sum test 

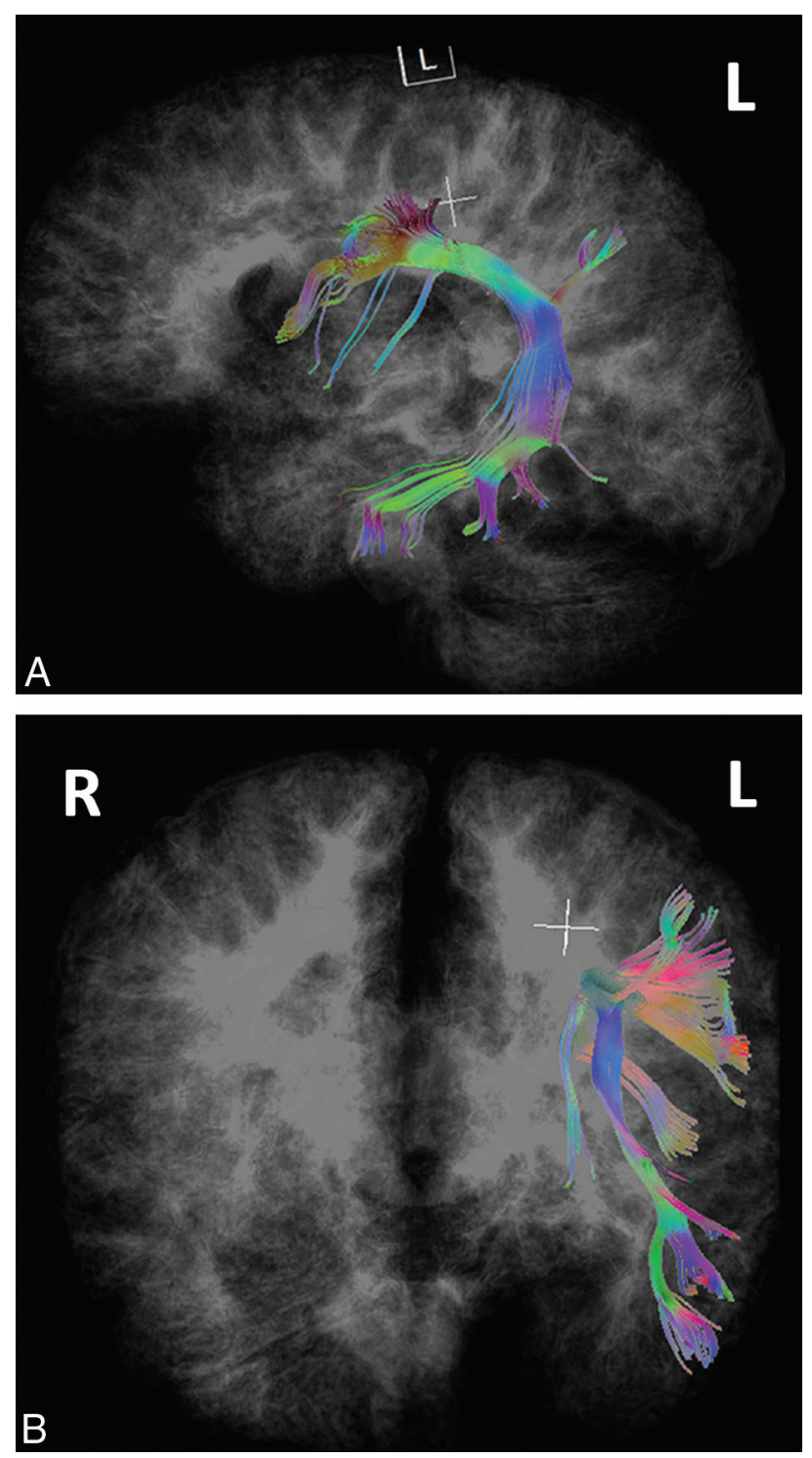

FIG 1. Left lateral $(A)$ and posterior $(B)$ views of a 3D tract reconstruction of the arcuate fasciculi in a control subject. Images demonstrate the presence of the arcuate fasciculus in the left hemisphere only. R indicates, right, L, left. The cursors reflect the center of the image space.
( $\alpha=.05$ ) was used to compare continuous variables (mean fractional anisotropy and mean diffusivity) between groups.

\section{RESULTS}

\section{Patients}

Imaging was performed from January 2009 to April 2011. Of the 49 patients with MCD identified as meeting the inclusion criteria, 1 was excluded on the basis of motion and 11 , on the basis of age (younger than 3 years). Thirty-seven patients with MCD (age range, 3-18 years; median, 10 years; 20 males, 17 females) and 22 age- and sex-matched controls (age range, 3-18 years; median, 10 years; 10 males, 12 females) composed the final study group. Cerebral malformations in the patient group included the following: polymicrogyria $(n=16)$, focal cortical dysplasia $(n=15)$, schizencephaly $(n=4)$, and gray-matter heterotopia $(n=2)$. Twentyone patients with MCD had intact language; 11 had mild-to-moderate impairment ( 4 with polymicrogyria, 4 with focal cortical dysplasia, 3 with schizencephaly); and 5 had profound impairment ( 3 with polymicrogyria, 1 with schizencephaly, 1 with focal cortical dysplasia).

\section{Left Arcuate Fasciculus}

All control subjects had intact language and an identifiable left arcuate fasciculus (see representative example in Fig 1). Similarly, all (21/21) patients with MCD with intact language had an identifiable left arcuate fasciculus (see example in Fig 2). By contrast, all (11/11) patients with MCD without an identifiable left arcuate fasciculus manifested some degree of language impairment (6 mild-to-moderate, 5 profound; see example in Fig 3). The frequency of language impairment in patients with MCD with no identifiable left arcuate fasciculus was significantly greater than that of those with a left arcuate fasciculus $(P<.001)$. Conversely, the frequency of the absence of the left arcuate fasciculus in patients with some degree of language impairment was significantly greater than that in patients with intact language $(P<.001)$. The diagnostic performance of an absent left arcuate fasciculus for some degree of language impairment is presented in Table 1. Although the patient number was insufficient to address statistically, all 4 left-handers with no identifiable left arcuate were impaired with respect to language.
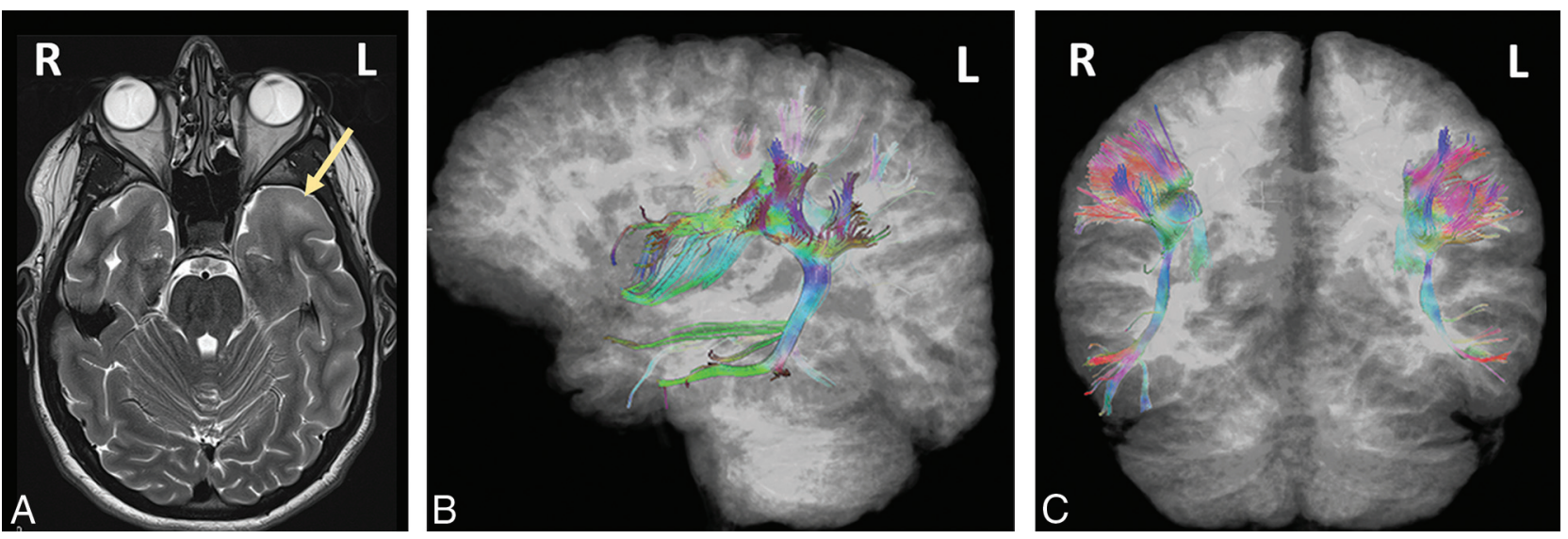

FIG 2. Axial T2-weighted image $(A)$ demonstrates focal cortical dysplasia (arrow) centered in the left anterior temporal lobe in a right-handed patient. Left lateral $(B)$ and posterior $(C)$ views of a 3D reconstruction demonstrate the appearance of the arcuate fasciculus in each hemisphere. This patient has normal language. 

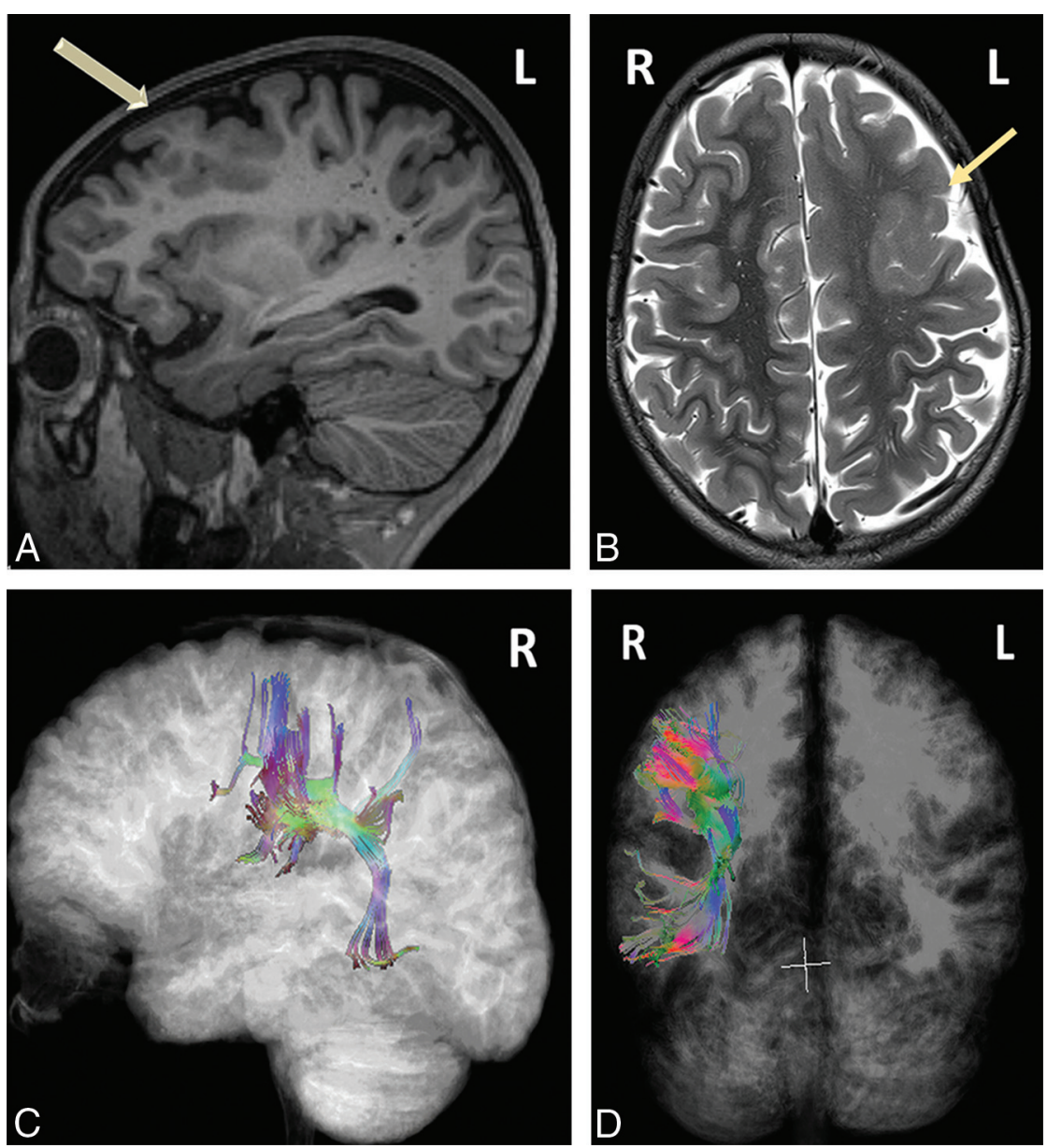

FIG 3. Sagittal MPRAGE $(A)$ and axial T2-weighted $(B)$ images demonstrate extensive focal cortical dysplasia (arrow) involving most of the visualized left frontal lobe. Right lateral $(C)$ and posterior $(D)$ views of the arcuate fasciculi demonstrate the presence of the arcuate fasciculus in the right hemisphere only. This right-handed patient was impaired with respect to language.
Table 1: Diagnostic performance of the absence of the left arcuate fasciculus with respect to language impairment

\begin{tabular}{lccc}
\hline & Diagnostic Performance & $\mathbf{9 5 \%}$ LCl & $\mathbf{9 5 \%} \mathbf{~ U C l}$ \\
\hline Sensitivity (\%) & 64.7 & 38.6 & 84.9 \\
Specificity (\%) & 100 & 78.1 & 100 \\
PPV (\%) & 100 & 67.8 & 100 \\
NPV (\%) & 75 & 52.9 & 89.3 \\
\hline
\end{tabular}

Note:- $-\mathrm{LCl}$ indicates lower limit of the $95 \%$ confidence interval; UCl, upper limit of the 95\% confidence interval; PPV, positive predictive value; NPV, negative predictive value.

Aside from its absence/presence, no significant differences in the character of the left arcuate fasciculus were observed when patients with MCD were compared with the control cohort. Specifically, there was no significant difference in tract diffusion metrics (either fraccuate fasciculi in patients with MCD versus controls. Trends toward higher fractional anisotropy in the left-versus-right arcuate fasciculus in both patients with MCD and controls did not meet statistical significance. There was no significant sex difference in tract metrics in either subject group, and the frequency of language impairment was not associated with sex.

\section{Right Arcuate Fasciculus}

Seventy-seven percent (17/22) of control subjects demonstrated an identifiable right arcuate fasciculus. Similarly, the right arcuate tional anisotropy or mean diffusivity) within the identifiable left ar- fasciculus was variably present in both the language-impaired (11/17) and language-intact $(15 / 20)$ patients with MCD. There was no difference in the sence in patients with MCD with versus without language impairment $(P=$ .722 ), and there was not a significant difference between the frequency of language impairment in patients with MCD with (10/26) versus without (7/ $11)$ a right arcuate fasciculus $(P=.283)$.

Of the 11 patients with MCD with absent left arcuate fasciculi, all of whom were language-impaired to some degree (see above), 4 also had no identifiable right arcuate fasciculus. All 4 of these patients with no identifiable arcuate fasciculus in either hemisphere were profoundly impaired with respect to language (see example in Fig 4). Of those remaining patients without a left arcuate fasciculus who had an identifiable right arcuate fasciculus, 6 were mild-to-moderately impaired, while only 1 was profoundly impaired. The frequency of profound language impairment among those with an absent left arcuate fasciculus was significantly greater in those patients whose right arcuate fasciculus was also absent $(P<.015)$. The diagnostic performance of failure to identify both the left and right arcuate fasciculi for profound language impairment is presented in Table 2. frequency of right arcuate fasciculus ab-

\section{DISCUSSION}

On the basis of this study in pediatric patients with MCD, we report 2 main findings: 1) Failure to identify the left arcuate fasciculus at diffusion tractography was universally associated with language impairment; and 2) in patients with no identifiable left arcuate fasciculus, failure to identify the right arcuate fasciculus was strongly associated with a complete absence of oral language.

Although incompletely understood, the fluent comprehension and production of language are best conceptualized as an emergent property that results from complex interaction between distributed cortical regions across the cerebrum. Traditionally, it was thought to be predicated primarily on Wernicke and Broca areas, located in the left posterior temporal and left inferior frontal lobes, respectively, interacting via the arcuate fasciculus. However, current understanding suggests that Wernicke and Broca areas are but a part of a richly interconnected, large-scale language network that extends to additional frontal, parietal, and temporal association areas in both hemispheres. ${ }^{29}$ Furthermore, optimal network function relies on interaction with cortical areas, primarily in the frontal and parietal lobes, which are not directly involved in language but rather facilitate cognitive function through working memory, attention, and other executive processes. ${ }^{21} \mathrm{~A}$ dualstream architecture of white matter pathways that promotes such 

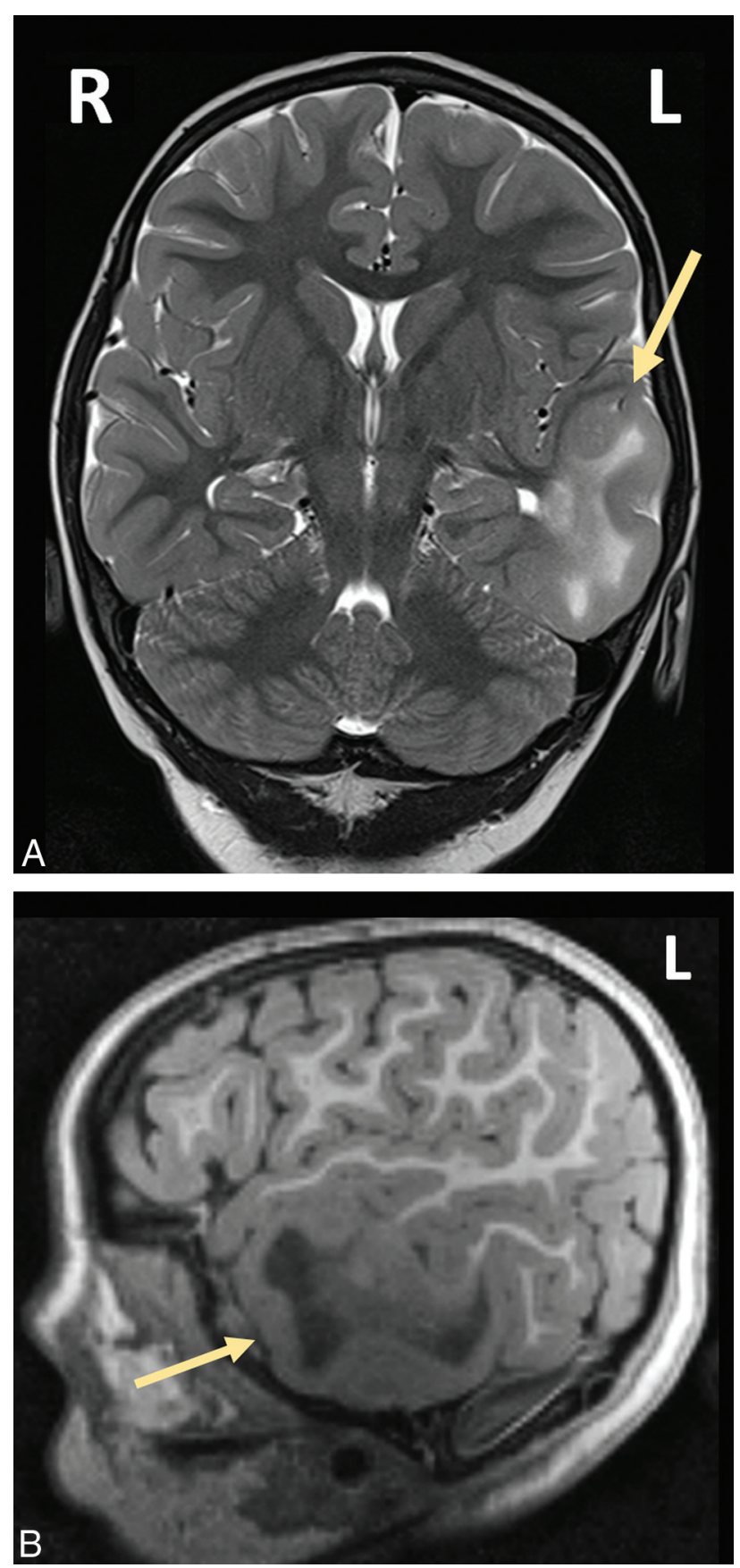

FIG 4. Coronal T2-weighted $(A)$ and sagittal MPRAGE $(B)$ images demonstrate a large focal cortical dysplasia (arrow) in the left temporal lobe. This patient has no identifiable arcuate fasciculus in either hemisphere (data not shown) and no language development.

cortico-cortical interaction and, therefore, subserves language function has been proposed. ${ }^{30}$ In general terms, the ventral stream is believed to link phonemic information with conceptual knowledge. Although the pathways that form the anatomic basis for this "stream" remain the subject of debate, roles for the uncinate, inferior longitudinal, and inferior fronto-occipital fasciculi have all been proposed. ${ }^{31}$ Specific functional attributes of the dorsal stream are less well-documented but generally are thought to involve the linkage between auditory and motor representations. $^{30}$ The arcuate/superior longitudinal fasciculus is generally considered to form the anatomic basis of the dorsal stream. ${ }^{32}$
Table 2: Diagnostic performance of the absence of both left and right arcuate fasciculi with respect to profound language impairment

\begin{tabular}{lccc}
\hline & Diagnostic Performance & 95\% LCI & 95\% UCI \\
\hline Sensitivity (\%) & 80 & 29.8 & 98.9 \\
Specificity (\%) & 100 & 86.7 & 100 \\
PPV (\%) & 100 & 39.6 & 100 \\
NPV (\%) & 96.9 & 82.4 & 99.8 \\
\hline
\end{tabular}

Note:- $\mathrm{LCl}$ indicates lower limit of the $95 \%$ confidence interval; $\mathrm{UCl}$, upper limit of the $95 \%$ confidence interval; PPV, positive predictive value; NPV, negative predictive value.

We found that failure to identify the left arcuate fasciculus at diffusion tractography was consistently associated with language dysfunction in our patient population. This finding highlights the importance of formation of the left arcuate fasciculus and, furthermore, suggests that it may be a prerequisite for normal language development. The right arcuate, by contrast, was variably identified in both patients and control subjects and was not clearly associated with language function in our cohort. However, in patients with no left arcuate, the inability to identify the right arcuate fasciculus was strongly associated with a complete failure to develop oral language. This finding suggests that the right arcuate fasciculus may, in fact, play an important role in language function, particularly in the setting of suboptimal development of more central network components. Together, our findings suggest the potential for diffusion tractography to provide clinically meaningful markers of network function in an individual patient. Absence versus presence of the arcuate fasciculus has recently been shown to be highly reliable; this finding adds to its appeal as a potential biomarker. ${ }^{25}$

Formation of association pathways involves numerous developmental processes that act in a coordinated fashion to establish mature patterns of cerebral connectivity. These include, but are not limited to, differentiation and maturation of pyramidal cells in cortical layer 3, axonal genesis and guidance to the subplate, synapse formation and establishment of connectivity with the developing cortical plate, and selection (or pruning) of connections. ${ }^{33}$ The last of these steps seems to involve activity-dependent stabilization of functionally useful synapses. ${ }^{34}$ Our observations suggest a frequent impact on the establishment and/or preservation of cortico-cortical connections in patients with MCD. One possible explanation for this finding is that such patients frequently exhibit abnormalities within the cortical layers involved in cortico-cortical connectivity. It would follow that abnormal cortical lamination could result in a diminished and/or abnormal contribution to the arcuate fasciculus and, therefore, failure to detect it at tractography. As an alternative explanation, disordered genetic regulation of molecular events involved in the formation of cortico-cortical connections could also account for the reported findings. In this scenario, absence of a detectable arcuate fasciculus need not be associated with histologic abnormality of any particular cortical layer.

The histopathologic significance of failure to identify a tract at tractography is yet to be established. In addition to the absence of the tract in an absolute sense, this finding could reflect marked disorganization of white matter structures, resulting in a profound loss of tissue coherence. As an additional possibility, research in epilepsy has demonstrated the presence of relatively 
widespread abnormal and epileptogenic networks in patients with focal structural lesions and, furthermore, has suggested the potential for ongoing seizure activity to establish and/or potentiate these networks. ${ }^{14,15,19}$ Aberrant connectivity resulting from such activity-dependent reorganization could also account for the findings in this study.

Although it has not been well-studied to date, the idea that cortico-cortical connections may be abnormal in patients with MCD is consistent with a report by Munakata et al, ${ }^{35}$ in which a case of unilateral left-sided polymicrogyria was associated with the absence of the left arcuate fasciculus. Similarly, Bernal et $\mathrm{al}^{36}$ reported the absence of the arcuate fasciculus in 2 cases of bilateral perisylvian polymicrogyria. More recently, Saporta et $\mathrm{al}^{24}$ suggested the functional relevance of such a finding after failing to identify the arcuate in 3 patients with congenital perisylvian syndrome and severe language dysfunction. Our results are also in line with work by Paldino et $\mathrm{al},{ }^{20}$ who used a machine-learning approach to predict language phenotype on the basis of wholebrain tractography data in a cohort of patients with epilepsy and MCDs. Specifically, they found that the left arcuate fasciculus was an important contributor to the language phenotype. Although the sensitivity for language impairment in our study was inferior to that attained by the machine, this difference might be explained, at least in part, by the access of the machine-learning algorithm to metrics related to all major white matter tracts in the left hemisphere and not only the arcuate.

In our study, the absence of the left arcuate fasciculus was highly specific for language impairment and, in our opinion, would be more easily generalized to clinical practice. The importance of the arcuate fasciculus to language function has also been suggested in other neurodevelopmental disorders. Abnormal, though identifiable, arcuate fasciculi have been reported in a range of disorders manifesting speech delay. ${ }^{37-39}$ Along similar lines, Wilson et $\mathrm{al}^{40}$ reported the absence of the left arcuate fasciculus in 6 of 7 patients with Angelman syndrome, a developmental disorder characterized by pervasive developmental delay and failure to develop speech. Sundaram et $\mathrm{al}^{41}$ reported the absence of the left arcuate fasciculus in 11 of 20 patients with global developmental delay. Both of the latter studies reported the presence of the left arcuate fasciculus in all healthy control subjects. These results are in line with ours and suggest that the absence of the left arcuate fasciculus may be a marker of language impairment in other patient populations. Of note, a study by Lebel and Beaulieu $^{42}$ reported a small frequency of the absence of the left arcuate fasciculus in healthy patients. In our control population, by contrast, all subjects had an identifiable left arcuate fasciculus. This discrepancy could be accounted for by the relatively small number of subjects in our study. Alternatively, it could reflect differences in image acquisition (including field strength and directional scheme for diffusion weighting) or methods of image processing. Further studies designed to rigorously define the normal character of the arcuate fasciculus at $3 \mathrm{~T}$ will be a necessary adjunct to future investigation.

This study has several limitations. First, it was a study of a selected cohort of patients with MCD. Extrapolation of these results to other patient groups at risk for language impairment may not be valid. Second, because fMRI was not performed, the actual locations of receptive and expressive language were not definitively ascertained. This limitation, however, is substantially mitigated by corroboration with language function. In particular, if there was a significant frequency of ectopic or right hemisphericdominant language function, it did not seem, in this study, to impact the prognostic significance of the imaging findings. Finally, functional assessment of language in this study was limited to a gross 3-point scale, chosen to reflect clinically relevant differences in language function while maximizing reproducibility of the assessment. Detailed neuropsychologic evaluation was not performed but would be of great potential value to future studies. In particular, such an evaluation might allow the identification of specific domains of language dysfunction in each patient, which could further elucidate functional subspecialization within the language network.

\section{CONCLUSIONS}

We report 2 main findings in pediatric patients with MCD: 1) Failure to identify the left arcuate fasciculus at diffusion tractography was consistently associated with language impairment; and 2 ) in patients with no left arcuate, failure to identify the right arcuate fasciculus was strongly associated with a complete absence of oral language. These findings suggest that the ability to form and/or maintain cortico-cortical connections is frequently impaired in patients with cortical malformations and, furthermore, is closely related to network function. In sum, our findings suggest the potential for diffusion tractography to provide clinically meaningful markers of network function in an individual patient.

\section{REFERENCES}

1. Basser PJ, Pajevic S, Pierpaoli $C$, et al. In vivo fiber tractography using DT-MRI data. Magn Reson Med 2000;44:625-32 CrossRef Medline

2. Melhem ER, Mori S, Mukundan G, et al. Diffusion tensor MR imaging of the brain and white matter tractography. AJR AmJ Roentgenol 2002;178:3-16 CrossRef Medline

3. Lee SK, Kim DI, Kim J, et al. Diffusion-tensor MR imaging and fiber tractography: a new method of describing aberrant fiber connections in developmental CNS anomalies. Radiographics 2005;25:5365, discussion 66-68 Medline

4. Catani M, Thiebaut de Schotten M. A diffusion tensor imaging tractography atlas for virtual in vivo dissections. Cortex 2008;44: 1105-32 CrossRef Medline

5. Vishwas MS, Chitnis T, Pienaar R, et al. Tract-based analysis of callosal, projection, and association pathways in pediatric patients with multiple sclerosis: a preliminary study. AJNR Am J Neuroradiol 2010;31:121-28 CrossRef Medline

6. Boyle CA, Decouflé P, Yeargin-Allsopp M. Prevalence and health impact of developmental disabilities in US children. Pediatrics 1994; 93:399-403 Medline

7. Cascino GD. Improving quality of life with epilepsy surgery: the seizure outcome is the key to success. Neurology 2007;68:1967-68 CrossRef Medline

8. Phi JH, Cho BK, Wang KC, et al. Longitudinal analyses of the surgical outcomes of pediatric epilepsy patients with focal cortical dysplasia. J Neurosurg Pediatr 2010;6:49-56 CrossRef Medline

9. Krsek P, Maton B, Jayakar P, et al. Incomplete resection of focal cortical dysplasia is the main predictor of poor postsurgical outcome. Neurology 2009;72:217-23 CrossRef Medline

10. Klein B, Levin BE, Duchowny MS, et al. Cognitive outcome of chil- 
dren with epilepsy and malformations of cortical development. Neurology 2000;55:230-35 CrossRef Medline

11. Lee SK, Kim DI, Mori S, et al. Diffusion tensor MRI visualizes decreased subcortical fiber connectivity in focal cortical dysplasia. Neuroimage 2004;22:1826-29 CrossRef Medline

12. Widjaja E, Blaser S, Miller E, et al. Evaluation of subcortical white matter and deep white matter tracts in malformations of cortical development. Epilepsia 2007;48:1460-69 CrossRef Medline

13. Widjaja E, Zarei Mahmoodabadi S, Otsubo H, et al. Subcortical alterations in tissue microstructure adjacent to focal cortical dysplasia: detection at diffusion-tensor MR imaging by using magnetoencephalographic dipole cluster localization. Radiology 2009; 251:206-15 CrossRef Medline

14. Galicia E, Imai K, Mohamed IS, et al. Changing ictal-onset EEG patterns in children with cortical dysplasia. Brain Dev 2009;31:569-76 CrossRef Medline

15. Jayakar $P$, Dunoyer $C$, Dean $P$, et al. Epilepsy surgery in patients with normal or nonfocal MRI scans: integrative strategies offer longterm seizure relief. Epilepsia 2008;49:758-64 CrossRef Medline

16. Jonas R, Asarnow RF, LoPresti C, et al. Surgery for symptomatic infant-onset epileptic encephalopathy with and without infantile spasms. Neurology 2005;64:746-50 CrossRef Medline

17. Jonas R, Nguyen S, Hu B, et al. Cerebral hemispherectomy: hospital course, seizure, developmental, language, and motor outcomes. Neurology 2004;62:1712-21 CrossRef Medline

18. Koh S, Mathern GW, Glasser G, et al. Status epilepticus and frequent seizures: incidence and clinical characteristics in pediatric epilepsy surgery patients. Epilepsia 2005;46:1950-54 CrossRef Medline

19. Lerner JT, Salamon N, Hauptman JS, et al. Assessment and surgical outcomes for mild type I and severe type II cortical dysplasia: a critical review and the UCLA experience. Epilepsia 2009;50:1310-35 CrossRef Medline

20. Paldino MJ, Hedges $K$, Zhang W. Independent contribution of individual white matter pathways to language function in pediatric epilepsy patients. Neuroimage Clin 2014;6:327-32 CrossRef Medline

21. Dronkers NF, Wilkins DP, Van Valin RD Jr, et al. Lesion analysis of the brain areas involved in language comprehension. Cognition 2004;92:145-77 CrossRef Medline

22. Yeatman JD, Dougherty RF, Rykhlevskaia E, et al. Anatomical properties of the arcuate fasciculus predict phonological and reading skills in children. J Cogn Neurosci 2011;23:3304-17 CrossRef Medline

23. Marchina S, Zhu LL, Norton A, et al. Impairment of speech production predicted by lesion load of the left arcuate fasciculus. Stroke 2011;42:2251-56 CrossRef Medline

24. Saporta AS, Kumar A, Govindan RM, et al. Arcuate fasciculus and speech in congenital bilateral perisylvian syndrome. Pediatr Neurol 2011;44:270-74 CrossRef Medline

25. Paldino MJ, Hedges K, Rodrigues KM, et al. Repeatability of quantitative metrics derived from MR diffusion tractography in paediatric patients with epilepsy. Br J Radiol 2014;87:20140095 CrossRef Medline

26. Im K, Paldino MJ, Poduri A, et al. Altered white matter connectivity and network organization in polymicrogyria revealed by individual gyral topology-based analysis. Neuroimage 2014;86: 182-93 CrossRef Medline

27. Basser PJ, Pierpaoli C. Microstructural and physiological features of tissues elucidated by quantitative-diffusion-tensor MRI. J Magn Reson B 1996;111:209-19 CrossRef Medline

28. Wakana S, Caprihan A, Panzenboeck MM, et al. Reproducibility of quantitative tractography methods applied to cerebral white matter. Neuroimage 2007;36:630-44 CrossRef Medline

29. Turken AU, Dronkers NF. The neural architecture of the language comprehension network: converging evidence from lesion and connectivity analyses. Front Syst Neurosci 2011;5:1 CrossRef Medline

30. Hickok G, Poeppel D. Dorsal and ventral streams: a framework for understanding aspects of the functional anatomy of language. Cognition 2004;92:67-99 CrossRef Medline

31. Dick AS, Tremblay P. Beyond the arcuate fasciculus: consensus and controversy in the connectional anatomy of language. Brain 2012; 135:3529-50 CrossRef Medline

32. Ellmore TM, Beauchamp MS, O’Neill TJ, et al. Relationships between essential cortical language sites and subcortical pathways. J Neurosurg 2009;111:755-66 CrossRef Medline

33. Friederici AD. Pathways to language: fiber tracts in the human brain. Trends Cogn Sci 2009;13:175-81 CrossRef Medline

34. Innocenti GM, Price DJ. Exuberance in the development of cortical networks. Nat Rev Neurosci 2005;6:955-65 CrossRef Medline

35. Munakata M, Onuma A, Takeo K, et al. Morphofunctional organization in three patients with unilateral polymicrogyria: combined use of diffusion tensor imaging and functional magnetic resonance imaging. Brain Dev 2006;28:405-09 CrossRef Medline

36. Bernal B, Rey G, Dunoyer C, et al. Agenesis of the arcuate fasciculi in congenital bilateral perisylvian syndrome: a diffusion tensor imaging and tractography study. Arch Neurol 2010;67:501-05 CrossRef Medline

37. Peters SU, Kaufmann WE, Bacino CA, et al. Alterations in white matter pathways in Angelman syndrome. Dev Med Child Neurol 2011;53:361-67 CrossRef Medline

38. Gopal SP, Tiwari VN, Veenstra AL, et al. Sensitive diffusion tensor imaging quantification method to identify language pathway abnormalities in children with developmental delay. J Pediatr 2012; 160:147-51 CrossRef Medline

39. Jeong JW, Sundaram SK, Kumar A, et al. Aberrant diffusion and geometric properties in the left arcuate fasciculus of developmentally delayed children: a diffusion tensor imaging study. AJNR Am J Neuroradiol 2011;32:323-30 CrossRef Medline

40. Wilson BJ, Sundaram SK, Huq AH, et al. Abnormal language pathway in children with Angelman syndrome. Pediatr Neurol 2011;44: 350-56 CrossRef Medline

41. Sundaram SK, Sivaswamy L, Makki MI, et al. Absence of arcuate fasciculus in children with global developmental delay of unknown etiology: a diffusion tensor imaging study. J Pediatr 2008;152: 250-55 CrossRef Medline

42. Lebel C, Beaulieu C. Lateralization of the arcuate fasciculus from childhood to adulthood and its relation to cognitive abilities in children. Hum Brain Mapp 2009;30:3563-73 CrossRef Medline 